\title{
Influência da autoconfiança materna sobre o aleitamento materno exclusivo aos seis meses de idade: uma revisão sistemática
}

\author{
Influence of maternal confidence on exclusive breastfeeding \\ until six months of age: a systematic review
}

Isabela Silva Rocha ${ }^{1}$

Luiz Fernando Lolli ${ }^{1}$

Mitsue Fujimaki ${ }^{1}$

André Gasparetto ${ }^{1}$

Najara Barbosa da Rocha ${ }^{1}$
${ }^{1}$ Programa de Residência em Odontologia em Saúde Coletiva e da Família, Departamento de Odontologia, Universidade Estadual de Maringá. Av Mandacaru 1550/Bloco S08, Jardim Paris. 87083170 Maringá PR Brasil. rochaisabelaa@gmail.com

\begin{abstract}
Breastfeeding is a practice directly related to the health of the mother and especially the baby. Despite being a natural process, many mothers report difficulties with breastfeeding. This study aimed to seek scientific evidence on the issue: "Can a mother with more confidence in breastfeeding exclusively breastfeed her baby for 06 months?". We conducted a systematic review with a search in five electronic databases, and included four cohort studies for evaluation. We conducted a qualitative analysis of the results, considering the methodological differences and lack of data, and were unable to perform meta-analysis. Confidence in breastfeeding was evaluated by using the Breastfeeding Self-Efficacy Scale. The studies analyzed showed statistically significant association between the practice of breastfeed and confidence in breastfeeding. The application of a scale to evaluate trust and identify mothers at risk for early weaning appeared to be a reliable measurement tool, capable of facilitating action planning by health professionals. The evidence showed association between mothers with higher levels of confidence in breastfeeding and exclusive breastfeeding for 6 months, but these results could not be generalized because of the heterogeneity of the population.
\end{abstract}

Key words Exclusive breastfeeding, Self-efficacy, Breastfeeding, Breastfeeding self-efficacy scale, Breastfeeding self-efficacy scale-short form
Resumo A amamentação é uma prática diretamente relacionada com a saúde da mãe e, principalmente, do bebê. Apesar de ser um processo natural, muitas mães relatam dificuldades. Este estudo objetivou buscar evidências científicas sobre a questão: "Mãe com mais confiança consegue amamentar exclusivamente por 6 meses?". Foi realizada uma revisão sistemática com busca em 5 bancos de dados eletrônicos, que resultou na inclusão de 4 estudos de coorte para avaliação. Realizou-se uma análise qualitativa dos resultados, porém não foi possivel fazer a meta-análise. Autoconfiança em amamentar foi avaliada pela escala "Breastfeeding Self-Efficacy Scale". Os estudos analisados mostraram que há associação estatística significante entre aleitamento materno exclusivo e autoconfiança em amamentar. A utilização da escala parece ser uma ferramenta confiável para identificar mães em risco para desmame precoce, facilitando o planejamento de ações pelos profissionais em saúde. As evidências obtidas mostraram a associação entre mães com maior confiança em amamentar e a amamentação exclusiva por 6 meses, porém estes resultados não podem ser generalizados devido à heterogeneidade das populações.

Palavras-chave Aleitamento materno, Autoeficácia, Amamentação, Breastfeeding self-efficacy scale, Breastfeeding self-efficacy scale-short form 


\section{Introdução}

A amamentação é uma prática de grande importância para o binômio mãe-filho, e que oportuniza a criação de vínculo e benefícios para a saúde de ambos. O aleitamento materno promove a involução genital no período pós-parto, diminui a chance de câncer de mama, ovário e útero, minimiza gastos com compra de produtos industrializados para alimentação da criança e com tratamentos para possíveis doenças. Em relação ao bebê, promove o desenvolvimento correto da face, fonação, respiração e deglutição e previne morbidade e mortalidade infantil. Alguns autores associam o aleitamento a um maior desenvolvimento intelectual com melhores níveis de escolaridade e de renda na idade adulta ${ }^{1-7}$.

Os órgãos internacionais e nacionais de saúde preconizam que a amamentação deve ser fonte de alimentação exclusiva para bebês, isto é, sem água e outros líquidos, até 06 meses de idade e após esse período pode-se complementar com outros alimentos sólidos e líquidos, mantendo a amamentação até dois anos de idade ou mais ${ }^{8}$ ${ }^{11}$. Mesmo com o início da amamentação exclusiva após o parto, muitas mães abandonam ou complementam esta prática logo nas primeiras semanas. Isso ocorre por diversos fatores, como: dificuldade do bebê na sucção dos seios, mamilos planos ou invertidos e/ou doloridos e produção insuficiente de leite ${ }^{2}$. Além destes, é possível citar: fatores emocionais da mãe, a condição socioeconômica, o grau de escolaridade, o incentivo da família, a real intenção de amamentar e também a falta de conhecimento sobre $o$ assunto pela mãe ou gestante $e^{3,6,12}$

Apesar da amamentação ser um prática natural e saudável, muitas mulheres relatam dificuldades, sendo estas influenciadas por diversos fatores, dentre eles: a autoconfiança da mãe. Estudos publicados recentemente descreveram que mães com menor autoconfiança, amamentam por menos tempo ${ }^{13,14}$.

De acordo com Bandura ${ }^{15}$, autoconfiança é confiança pessoal em realizar, de forma eficaz, uma tarefa ou alcançar um objetivo em especial. Em relação à amamentação, essa característica pode gerar "confiança ou expectativa positiva da mulher com relação aos seus conhecimentos e habilidades para amamentar seu bebê com êxito" ${ }^{16-21}$. Baseando-se nessas informações, um instrumento para a avaliação da autoconfiança materna foi desenvolvido e validado por Dennis e Faux ${ }^{16}$, intitulado Breastfeeding Self-EfficacyScale (BSES). Ele se fundamenta em quatro fontes de informação: experiência pessoal - positivas e/ou negativas sobre amamentação anterior; observação de outras experiências - conversar com mães que já amamentaram; persuasão verbal - incentivo e estímulo de pessoas próximas a ela e de entendedores sobre o assunto; e, por fim, fatores emocionais e fisiológicos - capazes de influenciar tanto positiva, quanto negativamente na realização da prática ${ }^{6,20,22,23}$. A escala foi traduzida e validada em diversos países, incluindo o Brasil, em dois modelos: 1) Escala de Autoeficácia na Amamentação original - Breastfedding Self-Efficacy Scale (BSES) ${ }^{4}$, contendo 33 itens e 2) Versão reduzida - Breastfeeding Self-Efficacy Scale-Short Form (BSES-SF), contendo 14 itens $^{24}$. A pontuação pode variar de 33 a 165 pontos na escala original ${ }^{16}$ e de 14 a $70^{17}$ na reduzida, indicando que quanto maior a pontuação, maior é a autoconfiança materna em amamentar, possibilitando aos profissionais de saúde reconhecer em quais situações a mulher apresenta menor confiança e intervir precocemente para evitar o desmame $^{6,16,17}$.

Observa-se que a autoconfiança em amamentar tem sido relatada como um fator preditivo para a duração e a exclusividade da prática de aleitamento materno $0^{3,21,25,26}$ e que a utilização da BSES é uma medida internacionalmente aplicável, confiável e válida para auxiliar os profissionais de saúde no cuidado de mulheres que amamentam ${ }^{4}$. No entanto, não há nenhuma revisão sistemática sobre a autoconfiança em amamentar e a prática de Aleitamento Materno Exclusivo (AME) aos 6 meses de vida do bebê.

Assim, o objetivo da presente revisão sistemática foi avaliar as evidências científicas relacionadas à seguinte questão: "Mãe com mais confiança para amamentar consegue amamentar exclusivamente por 06 meses?" Foi utilizada a técnica PICO (P = população, I = Intervenção, $\mathrm{C}=$ controle e $\mathrm{O}=$ "outcome" que signifca desfecho) para a formulação da pergunta da pesquisa, sendo: (1) População: mulheres gestantes e puérperas de primeira gestação ou não; (2) Intervenção/exposição a fatores de risco: A autoconfiança materna em amamentar avaliada por meio das escalas BSES e BSES-SF; (3) Desfecho: Aleitamento materno exclusivo até os 6 meses.

\section{Metodologia}

Buscou-se estudos longitudinais (coorte, casocontrole e ensaios clínicos) com mulheres e gestantes de todas as idades, que relataram sobre a 
prática do aleitamento materno exclusivo aos 6 meses de idade e a autoconfiança materna em amamentar. A seleção do estudo foi realizada em duas fases: (1) triagem de resumos e títulos e (2) leitura dos textos integrais selecionados para determinação do conjunto final da amostra.

A questão investigada foi: "Mulher (gestante ou puérpera) com maior autoconfiança em amamentar, amamenta exclusivamente por até 6 meses ou mais?".

\section{Estratégia de busca}

Os critérios de inclusão para a realização desta revisão sistemática foram: estudos longitudinais, ensaios clínicos, ensaios controle; estudos publicados entre 01 de janeiro de 1999 (data da primeira publicação sobre Breastfeeding Self-EficacyScale) a 01 de setembro de 2015; aplicação da escala BSES (original e reduzida); estudos que apresentam acompanhamento do aleitamento por 06 meses ou mais; que analisam a escala específica e também outras variáveis; encontrados apenas em revistas indexadas; com resultados; realizados com mulheres (primíparas ou não), sem distinção de idade ou raça e publicados nos idiomas português, inglês ou espanhol.

A pesquisa dos artigos foi realizada com a participação de dois revisores independentes (ISR, NBR), em 5 bases de dados: Medline do PubMed (http://www/pubmed.gov); Web of Science (http://www.isiknowledge.com); Cochrane Library (http://www.cochrane.org/index. htm); US National Institutes of Health (http:// www.clinicaltrials.gov) e Biblioteca Virtual em Saúde (Bireme, América Latina) (http : //www. bireme.br).

A estratégia de busca realizada no Medline, Web of Science e Cochrane Library foi: ("breast feeding" [Mesh] OR breastfeed ${ }^{\star}$ OR breast feed ${ }^{\star}$ OR breast-feed ${ }^{\star}$ OR breastfed OR breast fed OR breast-fed OR weaning) AND (breastfeeding self-efficacy scale OR breast feeding self-efficacy scale OR breast-feeding self-efficacy scale OR breastfeeding self efficacy scale OR breast feeding self efficacy scale OR breast-feeding self efficacy scale OR BSES OR breastfeeding confidence OR breast feeding confidence OR breast-feeding confidence OR BSES-SF) NOT ("animals"[Mesh] NOT “man" [Mesh])).

No US National Institutes of Health foi utilizada a combinação das seguintes palavras-chaves: "breast feeding", "breast feeding self efficacy scale”. Enquanto no Bireme, a combinação utilizada foi: “aleitamento materno", “auto- eficácia”, "questionário", "gravidez", “feminino”, “amamentação".

A busca online teve como resultado 3.810 títulos. Utilizou-se o programa Endnote Web (http://www.myendnoteweb.com/) para a organização dos estudos. Após a remoção das referências duplicadas, avaliou-se título/ resumo de 3.398 publicações, sendo que por volta de $10 \%$ destes foram lidos de forma independente por três revisores para determinação do acordo inter-examinador (Kappa: 0,71). As discordâncias foram resolvidas por consenso. Após a leitura, 3.271 registros foram excluídos por título/ resumo, uma vez que não cumpriam os critérios de inclusão estabelecidos.

Foram considerados critérios de exclusão: aleitamento materno exclusivo antes dos 6 meses; estudos que não apresentavam aplicação das escalas BSES e/ou BSES-SF; desfecho que não comparasse o escore da escala com a AME aos 6 meses; estudos com desenhos metodológicos diferentes de longitudinal (transversais, laboratoriais, relatos e séries de caso, de opinião); publicados antes de 1999; realizados em animais e/ ou homens; sem resultados ou não publicados.

Selecionou-se 127 publicações para análise do texto completo (Figura 1). Ensaios clínicos não foram encontrados. Executou-se um exercício de calibração com 06 estudos para, na sequência, realizar a leitura de todos os textos completos pelos dois revisores independentes (ISR, NBR).

\section{Extração de dados}

A extração de dados foi realizada pelos dois revisores (Figura 1), os quais tinham como desfecho de interesse: mães que apresentavam altas pontuações na aplicação da BSES e/ou BSES-SF, ou seja, maior autoconfiança, conseguiam assim, amamentar exclusivamente por mais tempo (no acompanhamento de 06 meses pós parto) do que aquelas que apresentavam pontuações menores.

\section{Avaliação de qualidade metodológica}

Para a avaliação da qualidade metodológica utilizou-se a Escala de Newcastle-Ottawa ${ }^{27}$ para estudos de coorte (Tabela 1), modificada de acordo com as necessidades expostas pelo presente estudo. A escala foi proposta por Wells, para avaliação de estudos não randomizados (estudos de coorte e de caso controle) e consiste em 3 dimensões: critérios para seleção da amostra, comparabilidade e resultados ${ }^{27}$. Cada dimensão é composta por oito itens que apresentam diversas 


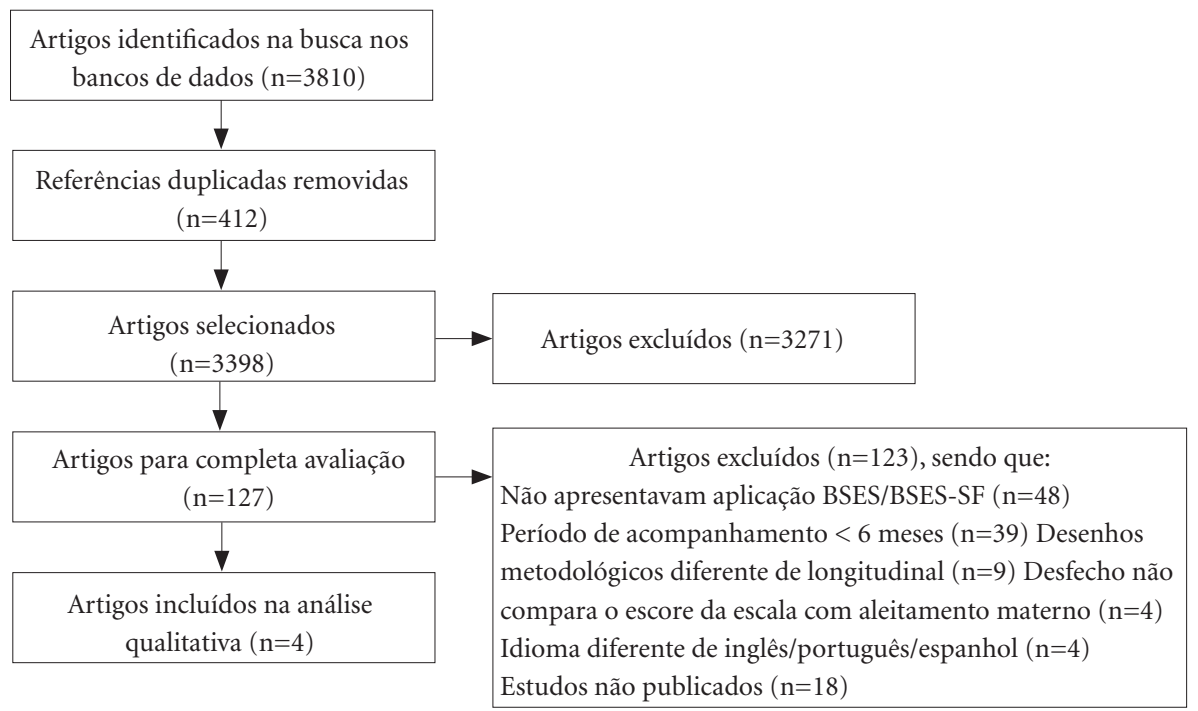

Figura 1. Fluxograma para a coleta de estudos, mostrando o número de estudos identificados, selecionados, elegíveis, e incluídos na revisão.

Tabela 1. Critérios de avaliação de qualidade utilizado para estudos de coorte por meio de uma versão modificada da escala Newcastle-Ottawa.

\section{Critérios}

Critérios para seleção da amostra

1) Representatividade da coorte exposta

(Autoconfiança em amamentar BSES

a) Amostra verdadeiramente representativa ${ }^{\star}$

b) Um pouco representativa da comunidade média (ex.: Hospital) *

c) Potencial para vieses de seleção ou não possui requisitos satisfatórios em parte (a)

d) Nenhuma descrição da derivação da coorte

2) Seleção do grupo não exposto

a) Tirado da mesma comunidade como coorte exposta ${ }^{*}$

b) Tirado de uma fonte diferente

c) Não há descrição de derivação da coorte não exposta

3) Verificação de exposição

a) Registro seguro (por exemplo, registros cirúrgicos) *

b) Entrevista estruturada com pesquisador calibrado *

c) Autorrelatório

d) Nenhuma descrição

4) Demonstração de que a autoconfiança em amamentar e a associação do AM estava presente no início do estudo
a) $\operatorname{Sim}$ *
$a\left(^{*}\right)$
$a\left(^{*}\right)$
$a\left(^{*}\right)$
$a\left(^{*}\right)$
b) Não

\section{McCarter-Spaulding Bosnjak et Ip et al., Henshaw} al., 2012

2012

et al., 2015 
Tabela 1. Critérios de avaliação de qualidade utilizado para estudos de coorte por meio de uma versão modificada da escala Newcastle-Ottawa.

\begin{tabular}{|c|c|c|c|c|}
\hline Critérios & $\begin{array}{l}\text { McCarter-Spaulding } \\
\text { \& Gore, } 2009\end{array}$ & $\begin{array}{l}\text { Bosnjak et } \\
\text { al., } 2012\end{array}$ & $\begin{array}{l}\text { Ip et al., } \\
2012\end{array}$ & $\begin{array}{l}\text { Henshaw } \\
\text { et al., } 2015\end{array}$ \\
\hline \multicolumn{5}{|l|}{ Comparabilidade } \\
\hline \multicolumn{5}{|l|}{$\begin{array}{l}\text { 1) A comparabilidade das coortes na base do } \\
\text { desenho ou análise }\end{array}$} \\
\hline $\begin{array}{l}\text { a) A exposição de interesse (BSES e } \\
\text { amamentação) é ajustada para um fator de } \\
\text { confusão * }\end{array}$ & $a\left(^{*}\right)$ & $a\left(^{*}\right)$ & $a\left(^{*}\right)$ & $a\left(^{*}\right)$ \\
\hline $\begin{array}{l}\text { b) A exposição de interesse (BSES e } \\
\text { amamentação) é ajustado para dois ou mais } \\
\text { fatores de confusão }\end{array}$ & & & & \\
\hline $\begin{array}{l}\text { (c) Descrição relacionada com a análise de ajuste } \\
\text { para fatores de confusão }\end{array}$ & & & & \\
\hline \multicolumn{5}{|l|}{ Resultado } \\
\hline \multicolumn{5}{|l|}{ 1) Avaliação do resultado } \\
\hline \multicolumn{5}{|l|}{ a) avaliação cega independente * } \\
\hline b) autorrelatório & $b$ & $b$ & $b$ & $b$ \\
\hline \multicolumn{5}{|l|}{ c) nenhuma descrição } \\
\hline \multicolumn{5}{|l|}{$\begin{array}{l}\text { 2) Acompanhamento por tempo suficiente para que } \\
\text { ocorressem resultados }\end{array}$} \\
\hline $\begin{array}{l}\text { a) Sim (período de acompanhamento de } 06 \\
\text { meses)* }\end{array}$ & $a\left(^{*}\right)$ & $a\left(^{*}\right)$ & $a\left(^{*}\right)$ & $a\left(^{*}\right)$ \\
\hline \multicolumn{5}{|l|}{ b) Não } \\
\hline \multicolumn{5}{|l|}{ 3) Adequação de acompanhamento de coortes } \\
\hline \multicolumn{5}{|l|}{ a) Completa - todos os sujeitos responderam * } \\
\hline $\begin{array}{l}\text { b) Indivíduos perdidos para followup improvável } \\
\text { para introduzir viés - número pequeno perdido } \\
(>10 \%)^{\star}\end{array}$ & $b\left(^{*}\right)$ & $b\left(^{*}\right)$ & $b\left(^{*}\right)$ & \\
\hline $\begin{array}{l}\text { c) Acompanhar taxa }<30 \% \text { e nenhuma descrição } \\
\text { daqueles perdida }\end{array}$ & & & & $c$ \\
\hline \multicolumn{5}{|l|}{ d) Nenhuma declaração } \\
\hline Total de estrelas & $6 / 9$ & $6 / 9$ & $6 / 9$ & $5 / 9$ \\
\hline
\end{tabular}

Um ponto $\left(^{*}\right)$; dois pontos $\left.{ }^{* *}\right)$; Pontuação Máxima: 9 pontos.

opções de respostas. Para realização da avaliação utilizou-se um sistema de estrela, no qual uma estrela correspondia a um ponto. Cada item recebeu uma estrela, com exceção do item "comparabilidade" que poderia receber duas. Desta forma a pontuação variou de zero a nove pontos, considerando-se menor ou maior qualidade, respectivamente ${ }^{27}$. As discordâncias foram resolvidas por consenso.

\section{Síntese dos dados}

As informações apresentadas pelos estudos analisados eram heterogêneas, já que variaram de acordo com o período em que a escala foi aplicada (de 48 horas a uma semana pós parto) e/ ou alguma diferença na característica da amostra (aplicação em mães primíparas ou em uma só etnia). Todavia, os resultados dos estudos não foram avaliados quantitativamente, porque não havia dados suficientes para serem agrupados, não sendo possível assim, a realização da meta-análise (Tabela 2).

\section{Resultados}

\section{Características dos estudos}

Entre os 127 trabalhos selecionados para a análise de texto completo, 04 estudos de coorte foram incluídos na presente revisão sistemáti- 
ca. Os trabalhos selecionados foram estudos de coorte realizados em países como Estados Uni$\operatorname{dos}^{19,28}$, China ${ }^{29}$ e Croácia ${ }^{26}$. Todos relacionaram, de alguma forma, a autoconfiança da mãe (medida por meio da aplicação da BSES ou BSES-SF) com o tempo de duração do aleitamento materno exclusivo aos 06 meses de idade. $\mathrm{O}$ aleitamento materno foi avaliado longitudinalmente desde o nascimento e em intervalos de tempo específicos até o final do estudo, no qual obteve-se o resultado.

\section{Análise Qualitativa}

\section{Fatores relacionados à duração} do aleitamento materno exclusivo

De acordo com os estudos analisados, um dos principais fatores relacionados à duração do aleitamento materno exclusivo foi a autoconfiança materna em amamentar. Também foram citados como importantes fatores: o ajustamento psicológico, imagem corporal, etnia e/ou raça, intenção da mãe em amamentar, idade, nível de escolaridade, se estava empregada, se vivia em casa própria, se morava com a família e/ou amigos; além de fatores emocionais ou sintomas depressivos, senso de coerência materno (capacidade da mãe em lidar com situações difíceis ou inesperadas em relação ao aleitamento materno) e se eram primíparas ou multíparas ${ }^{19,26,28,29}$.

\section{Escore da BSES/ BSES-SF e a duração do aleitamento materno exclusivo}

Todos os estudos enfatizaram a informação de que mães que apresentaram maior pontuação na aplicação da BSES/BSES-SF, amamentaram exclusivamente por mais tempo (no acompanhamento de 06 meses pós-parto). Contudo, alguns estudos demonstraram possíveis falhas nesse quesito, uma vez que a pontuação apresentada por mulheres primíparas e multíparas foi relativamente diferente. Acredita-se que aquelas que já tiverem mais de um filho possuem experiência prévia em aleitamento materno e, desta forma, sua resposta em relação à confiança em amamentar é consequentemente diferente daquelas que não tiveram essa mesma vivência, já que se tratava da primeira gravidez ${ }^{19,26,29}$.

\section{Avaliação da Qualidade}

A avaliação da qualidade foi realizada por meio da escala de Newcastle-Ottawa modificada, e obteve-se uma pontuação entre 5 e 6 (sendo o máximo 09 pontos). As principais deficiências 
foram relacionadas ao processo de coleta de dados, pois os estudos não apresentavam o mesmo padrão na população analisada, já que alguns foram realizados apenas com mães primíparas ${ }^{28}$ enquanto outros com amostras contendo também mães multíparas ${ }^{19,26,29}$. Todos foram realizados por meio de autorrelatório, ou seja, questionários autoaplicados.

\section{Resultados dos estudos em relação aos valores de autoconfiança e a prática do aleitamento materno exclusivo aos 06 meses}

As análises dos valores de autoconfiança apresentados pelas mães, por meio dos escores obtidos nas escalas aplicadas nos estudos, mostraram diferenças significativas na pontuação, já que não houve uma padronização do tipo da escala utilizada. Assim, o estudo que utilizou a escala em sua forma completa não apresentou valores de aplicação da escala BSES, por este não ser o objetivo do estudo ${ }^{28}$. Ainda, os estudos que apresentaram a aplicação da escala em sua forma reduzida, tiveram os valores médios: $41,1^{29} ; 51,9^{19}$ e $55^{26}$. Dentre os estudos, 03 mostraram que houve uma relação significante entre a pontuação obtida e a duração do aleitamento materno exclusivo, sendo que a eficácia materna aumentou com o tempo de AME em 06 meses; somente o estudo de Ip et al. ${ }^{29}$ não deixou claro o valor da associação (valor de p), porém foi relatado que a associação foi significante.

\section{Relação entre fatores demográficos e a duração do aleitamento materno exclusivo}

No estudo de Bosnjack et al. ${ }^{26}$, os fatores demográficos idade materna e alto nível de escolaridade tiveram associação positiva com o tempo do aleitamento materno exclusivo. Neste estudo também foi feita a associação entre a BSES-SF e a capacidade da mulher em lidar com situações estressantes durante o período de amamentar (SOC), que se mostrou positiva, já que mães com SOC positivos construíram ou melhoraram sua autoeficácia em amamentar.

Já Ip et al. ${ }^{29}$ e Henshaw et al. $^{28}$ não fizeram esta relação em seus estudos, contudo o último demonstrou que altas pontuações da BSES estiveram relacionadas à menores sintomas de depressão e maior ajustamento emocional nas mães em período pós-parto.

Ainda, McCarter-Spaulding e Gore ${ }^{19}$ relataram que não houve diferença entre a pontuação da escala e a idade materna, estado civil e renda familiar das mães. Entretanto, houve diferenças com base na etnia, no qual mulheres afro-americanas atingiram um valor menor do que aquelas que não se consideravam como africanas.

\section{Discussão}

\section{Objetivos e Fatores particulares relacionados ao aleitamento materno exclusivo envolvidos nos estudos analisados}

Os objetivos apresentados por cada estudo foram heterogêneos, no entanto todos avaliaram a realização da amamentação e uma das escalas BSES/BSES-SF foram aplicadas. Bosnjack et al. ${ }^{26}$ e Ip et al. ${ }^{29}$ objetivaram traduzir e validar a escala na forma reduzida em mulheres da Croácia e em Hong Kong no Japão, respectivamente, além de avaliar a capacidade da mesma em identificar mães susceptíveis ao desmame precoce. Diferentemente, Henshaw et al..$^{28}$ pretenderam avaliar prospectivamente a relação entre o humor e os resultados da amamentação em mães primigestas. McCarter-Spaulding e Gore ${ }^{19}$ examinaram as propriedades psicométricas da BSES-SF em mulheres negras dos Estados Unidos.

E em vista disso, houve particularidades importantes a serem destacadas nesta revisão sistemática, como o fato de que mães com experiência anterior na amamentação em período maior que seis meses, apresentaram índices mais altos de autoeficácia ${ }^{19,26}$. Ip et al. ${ }^{29}$ e Henshaw et al. ${ }^{28}$ concordam que o AME diminui até os 06 meses de idade. Enquanto que McCarter-Spaulding e Gore $^{19}$ descreveram que a autoeficácia em amamentar pode variar, de acordo com a cultura ou etnia das mulheres negras, indicando que as abordagens educativas devem ser realizadas de forma apropriada para essas mulheres. Portanto, pode-se perceber em todos os estudos que a escala BSES (em suas duas variações) foi um instrumento efetivo para previsão da duração do aleitamento materno exclusivo, em mães primíparas ou não, e também na identificação daquelas que se encontram em risco de desmame antes dos seis meses. Ademais, mostraram que é importante que os profissionais de saúde usem essa ferramenta para detectar mães com risco de desmame precoce quando programas educacionais forem desenvolvidos sobre a amamentação e que também estimulem as mães a ter uma maior percepção sobre seu desempenho na prática, resultando em aumento de sua confiança em amamentar. 


\section{Potenciais viéses no processo desta revisão}

Não houve viés relacionado ao ano de publicação, porque a escala foi desenvolvida a partir de 1999 e, por isso, não há nenhum estudo antes deste período. A seleção envolveu artigos publicados entre 1999 e 2015. Todos os estudos analisados foram publicados em Inglês, no entanto um viés em relação ao idioma foi encontrado no processo de seleção dos artigos: quatro publicações foram previamente selecionadas, mas não foram incluídas na avaliação final por estarem escritas em idiomas diferentes dos quais eram previstos nos critérios de inclusão.

Um fator de confusão encontrado foi a falta de artigos que analisassem a autoeficácia em amamentar e a prática de AME como principal desfecho, sem a presença de outros objetivos no estudo.

Além disso, a escala aplicada no período pós -parto também pode corresponder a um viés, pois a sua aplicação difere na primeira gestação e em mães que já experimentaram a prática do aleitamento materno, visto que nessa fase a mãe já passa a ter uma percepção maior sobre ela, o que, consequentemente, interfere em sua autoconfiança.

Um possível viés encontrado foi o fato de que os estudos não deixavam claro os resultados dos escores da escala com a associação do AME aos 06 meses de idade. Alguns avaliaram somente a média dos escores da amostra enquanto outros não apresentavam nenhum dado sobre o escore. Essas informações seriam de extrema importância para uma avaliação mais detalhada da autoconfiança, tornando possível a realização da meta-análise e melhorando a evidência dos resultados.

Ainda poderia ser considerado como viés, a diminuição da amostra no decorrer do seguimento do estudo, fato que pode diminuir sua validade, principalmente aqueles que apresentaram perda maior que $30 \%$.

A não padronização de tempo específico para aplicação da escala, das pesquisas incluídas nesta revisão, também foi vista como um viés, por não permitir identificar qual o período mais indicado para aplicar a escala e reconhecer as mães com risco de abandonar precocemente a prática do aleitamento exclusivo. Houve variação de dias, entre dois e sete dias de puerpério e essa pode interferir na obtenção dos resultados porque, como já relatado nesta revisão, o contato da mãe com o aleitamento e a experiência obtida nesse período interfere de forma clara e expressiva na percepção dela sobre a execução da prática e em sua autoconfiança. É válido ressaltar que o período apontado nesta revisão (de 2 a 7 dias) é favorável para a aplicação destas escalas, sendo que em todos os estudos houve uma associação positiva entre o nível de confiança e AME aos 6 meses.

Os resultados não podem ser generalizados a toda população, devido as particularidades e heterogeneidade metodológica de cada estudo incluído nesta revisão.

\section{Avaliação da qualidade dos estudos incluídos}

Não foi possível a realização da meta-análise dos resultados coletados neste estudo. Os artigos avaliados não disponibilizaram os dados estatísticos suficientes para sua execução, como por exemplo: p-valor, desvio padrão, intervalo de confiança, entre outros. Apesar disso, com os resultados descritos e a análise da discussão nos estudos, verificou-se que a autoconfiança é um fator que influencia significativamente a prática de aleitamento materno exclusivo em 06 meses pós-parto.

Durante o seguimento de estudos de coorte a perda de amostra aceitável é de até $10 \%$, porque se tornam comuns devido a mudanças de endereço e/ou telefone, desinteresse em participar, adoecimento e, até mesmo, morte ${ }^{30}$. Viés de amostra foi visto em apenas um dos estudos, o qual apresentou uma perda de amostra maior que $30 \%{ }^{28}$.

Em 03 estudos percebeu-se que mais de 60\% de mães conseguiram amamentar até os 06 meses, enquanto outro apresentou um percentual menor de 30\% de mães que conseguiram a realização da prática exclusiva até o período pretendido $^{19,28}$. A baixa prática de AME até os 06 meses de idade acontece nacionalmente e mundialmente já que vários fatores interferem no processo, inclusive crenças, que devem ser desmotivadas pelos profissionais de saúde.

Outro problema apresentado foi o viés de informação. Em alguns dos estudos, a BSES/ BSES-SF foi aplicada em mães que já tiveram experiências de sucesso ou fracasso anterior em amamentar, podendo, na maioria das vezes, demonstrar uma autoconfiança maior e, consequentemente, uma pontuação significantemente maior do que as mães que não possuíam essa experiência prévia ${ }^{19,26,29}$.

Viés de seleção também foi uma falha apresentada por alguns estudos, nos quais as amostras eram compostas por mães de nacionalidades diferentes ou mães com níveis mais altos de esco- 
laridade (ex.: análise de mulheres apenas com ensino superior completo) ou mães identificadas de acordo com a etnia. Houve casos em que o autor descreveu que a amostra analisada foi insuficiente para a realização de comparação entre os grupos, porque os dados obtidos por meio da média dos escores da aplicação da escala foram insuficientes para obtenção do resultado desejado ${ }^{19,28}$.

Os autores que desenvolveram as escalas BSES e BSES-SF ${ }^{16,17}$ propuseram que estas fossem autoaplicadas e, por isso, o tipo de inquérito realizado por todos os estudos desta revisão se apresentou como autorrelatório. Mesmo assim, na escala qualitativa proposta por este estudo optou-se em permanecer com a falta de qualidade para este quesito, já que sabe-se que pode levar ao excesso ou a falta de informação sobre o aleitamento materno influenciando na correta avaliação dos dados ${ }^{26}$.

\section{Justificação para a exclusão}

De acordo com Burns ${ }^{31}$, o estudo de coorte apresenta o mais alto nível de evidência e é menos propenso à apresentação de viéses. Um pequeno número de estudos de coorte foi encontrado e incluído no presente estudo. Isto se deve em razão de que uma minoria aplicou a escala BSES/BSES-SF, que é o principal objeto de avaliação do presente trabalho, da mesma forma, como o período de acompanhamento de 06 meses de aleitamento materno exclusivo preconizado pelas Organizações Mundiais de Saúde. Deste modo, os estudos que não possuíam esses dois fatores foram excluídos por não permitirem a realização da avaliação da autoconfiança da mãe em amamentar.

A ausência de resultados também foi um fator de exclusão de estudos, igualmente aqueles que não apresentaram a relação entre o escore da escala com o aleitamento materno e idioma diferente de inglês, português e espanhol.

\section{Nível de evidência}

As evidências obtidas nesta revisão sistemática verificaram que mães que apresentam alto índice de confiança no ato de amamentar, mantêm exclusivamente o aleitamento materno por mais tempo em relação àquelas que apresentam escores menores. Contudo, não foi possível confirmar se a aplicação da BSES/BSES-SF no período de pós-parto influenciou a obtenção de escores maiores, já que se acredita que a experiência obtida pelas mães com a prática em amamentar pode, mesmo sendo pouca, influenciar positiva ou negativamente na prática do aleitamento materno exclusivo, resultando na obtenção de escores diferentes do que aqueles que seriam obtidos se a escala fosse aplicada no período pré-natal.

Houve heterogeneidade na metodologia aplicada nos estudos e falta de avaliação cega, visto que a maioria apresentou a realização do autorrelatório, fato proeminente para ocorrência de viéses de informação. Ademais, a ausência de controle para fatores de confusão, como variáveis psicológicas e autoeficácia em amamentar no período pré-natal, que também interferem na prática do aleitamento exclusivo devem ser apontados. A não inclusão de mães multíparas na amostra seria uma boa estratégia para os próximos estudos.

As evidências relatadas nesta revisão são atualmente a compilação de resultados mais recentes para guiar uma conduta clínica sobre a identificação de mães para o desmame precoce, mesmo com suas limitações, até que novos estudos de coorte observacionais ou experimentais sejam realizados. Vale salientar que não foram encontrados estudos clínicos randomizados de boa qualidade sobre o assunto para apoiar as conclusões, e que esta carência deve ser suprida para se obter melhores resultados entre autoconfiança ao amamentar e a prática de aleitamento materno exclusivo aos 06 meses de idade.

\section{Conclusão}

Os resultados dos estudos que constituem esta revisão sistemática demonstraram que a autoconfiança da mulher esta associada com a duração mais duradoura do aleitamento materno exclusivo, porém não é possível a generalização dos resultados devido a heterogeneidade das populações estudadas.

É interessante salientar a importância do profissional da saúde em reconhecer a autoeficácia materna (sendo primíparas ou não) e desenvolver estratégias individualizadas para fortalecer ou, até mesmo, construir a confiança das mesmas para a realização da prática de amamentar de maneira correta e exclusiva, visto que, muitas vezes, a baixa confiança pode estar relacionada com ajustes emocionais como, por exemplo, a depressão pós parto e, consequentemente, a ocorrência do desmame precoce.

Houve o reconhecimento de que as escalas BSES e BSES-SF podem ser uma ferramenta de medida prática, válidas e confiáveis da autoeficá- 
cia das mães, que podem estar em risco para a interrupção prematura do aleitamento materno exclusivo. Além do mais, sua utilização pode ser grande facilitador para as atividades dos profissionais em saúde.

\section{Diretrizes para futuras pesquisas}

Estudos de coorte e ensaios clínicos randomizados com dados longitudinais devem ser realizados sobre a relação entre a autoeficácia em amamentar e a duração do aleitamento materno exclusivo. Torna-se necessária a realização de pesquisas no período pré-natal a fim de identificar mães com risco precoce de abandono da prática do aleitamento exclusivo.

\section{Colaboradores}

IS Rocha - Coleta, Interpretação e análise dos dados, preparação e redação do manuscrito revisão crítica e aprovação final; NB Rocha - Concepção e delineamento do estudo, orientação do estudo, interpretação e análise dos dados, preparação e redação do manuscrito revisão crítica e aprovação final; M Fujimaki, LF Lolli e A Gasparetto Interpretação e análise dos dados, revisão crítica e aprovação final.

\section{Agradecimentos}

Ao Programa de Residência em Odontologia em Saúde Coletiva e da Família do Departamento de Odontologia da UEM pelo apoio ao projeto e ao Hospital Universitário Regional de Maringá UEM pela concessão da bolsa de residência. 


\section{Referências}

1. Bueno LGDS, Teruya KM. Aconselhamento em amamentação e sua prática. J Pediatr 2005; 80(5):126-130.

2. Brasil. Ministério da Saúde (MS). Aleitamento materno. Brasília: MS; 2009.

3. McCarter-Spaulding DE, Dennis CL. Psychometric testing of the Breastfeeding Self-Efficacy Scale-Short Form in a sample of Black women in the United States. Res Nurs Health 2010; 33(2):111-119.

4. Zubaran C, Foresti K, Schumacher M, Thorell MR, Amoretti A, Müller L, Dennis CL. The Portuguese version of the Breastfeeding Self-Efficacy Scale-Short Form. JHL 2010; 26(3):297-303.

5. Moimaz SAS, Rocha NB, Garbin AJI, Saliba O. Relação entre aleitamento materno e hábitos de sucção não nutritivos. Cien Saude Colet 2011; 16(5):2477-2484.

6. Marmotti E, Epifanio M. Aleitamento materno exclusivo e a Escala de Autoeficácia na Amamentação. Rev Rene 2014; 15(5):771-779.

7. Victoria CG, Horta BL, Mola CL, Quevedo L, Pinheiro RT, Gigante DP, Gonçalves H, Barros FC. Association between breastfeeding and intelligence, educational attainment, and income at 30 years of age: a prospective birth cohort study from Brazil. Lancet Glob Health 2015; 3(4):e199-e205.

8. Organização Mundial da Saúde (OMS). Evidências científicas dos dez passos para o sucesso no aleitamento materno. Brasília: Organização Pan-Americana da Saúde; 2001.

9. Brasil. Constituição da República Federativa do Brasil de 1988. Diário Oficial da União 1988; 5 out.

10. World Health Organization (WHO). Exclusive breastfeeding for six months best for babies everywhere. Geneva: WHO; 2011.

11. Brasil. Lei no 11.770 , de 9 de setembro de 2008. Cria o Programa Empresa Cidadã, destinado a prorrogação da licença-maternidade mediante concessão de incentivo fiscal, e altera a Lei no 8.212, de 24 de julho de 1991. Pub. L. no 11.770. Diário Oficial da União 2008; 9 set.

12. Rocha NB, Garbin AJI, Garbin CAS, Moimaz SAS. O ato de amamentar: um estudo qualitativo. Physis Saúde Coletiva 2010; 20(4):1293-1305.

13. Melo Dodt RC, Ferreira AMV, Nascimento LA, Macêdo AC, Joventino ES, Ximenes LB. Influência da Estratégia de Educação em Saúde Mediada por Álbum Seriado Sobre a Autoeficácia Materna para Amamentar. Texto Contexto Enferm 2013; 22(3):610-618.

14. Rocha NB, Garbin AJI, Garbin CAS, Saliba O, Moimaz SAS. Estudo Longitudinal sobre a Prática de Aleitamento Materno e Fatores Associados ao Desmame Precoce. Pesq Bras Odontoped Clin Integr 2013; 13(4):337-342.

15. Bandura A. Self-efficacy: Toward a unifying theory of behaviour change. Psychological Review 1077; 84(2):191-215.

16. Dennis C, Faux S. Development and psychometric testing of the Breastfeeding Self-Efficacy Scale. Res Nurs Health 1999; 22(5):399-409.

17. Dennis C. The breastfeeding self-efficacy scale: psychometric assessment of the short form. J. Obstet Gynaecol Neonatal Nurs 2003; 32(6):734-744.

18. Dennis CE. Identifying predictors of breastfeeding self-eficacy in the immediate postpartum period. Res Nurs Health 2006; 29(4):256-268.
19. McCarter-Spaulding D, Gore R. Breastfeeding Self-Efficacy in Women of African Descent. JOGNN 2009; 38(2):230-243.

20. Rodrigues AP, Padoin SMM, Guido LA, Lopes LFD. Fatores do pré-natal e do puerpério que interferem na autoeficácia em amamentação. Esc. Anna Nery Rev Enferm 2014; 18(2):257-261.

21. Jager E, Broadbent J, Fuller- Tyszkiewics M, Nagle C, McPhie S, Skouteris H. A longitudinal study of the effect of psychosocial factors on exclusive breastfeeding duration. Midwifery 2015; 31(1):103-111.

22. Blyth R, Creddy DK, Dennis CL, Moyle W, Pratt J, De Vries SM. Effect of maternal confidence on breastfeeding duration: an application of breastfeeding self-efficacy theory. Birth 2002; 29(4):278-284.

23. Tavares MC, Aires JS, Melo Dodt RC, Joventino ES, Oriá MOB, Ximenes LB. Application of Breastfeeding Self-Efficacy Scale-Short Form to post-partum women in rooming-in care: a descriptive study. Online Braz J Nurs 2010; 9(1):1-13.

24. Oriá MOB, Ximenes LB, de Almeida BC, Glick DF, Dennis CL. Psychometric Assessment of the Brazilian Version of the Breastfeeding Self-Efficacy Scale. Public Health Nurs 2009; 26(6):574-583.

25. Wutke K, Dennis C. The reliability and validity of the Polish version of the Breastfeeding Self-Efficacy ScaleShort Form: Translation and psychometric assessment. Int J Nurs Stud 2007; 44(8):1439-1446.

26. Pavicic Bosnjak A, Rumboldt M, Stanojevic M, Dennis CL. Psychometric Assessment of the Croatian Version of the Breastfeeding Self-Efficacy Scale-Short Form. JHL 2012; 28(4):565-569.

27. Stang A. Critical evaluation of the Newcastle-Ottawa scale for the assessment of the quality of nonrandomized studies in meta-analyses. Eur J Epidemiol 2010; 25(9):603-605.

28. Henshaw EJ, Fried R, Siskind E, Newhouse L, Cooper M. Breastfeeding Self-Efficacy, Mood, and Breastfeeding Outcomes among Primiparous Women. JHL 2015; 31(3):511-518.

29. Ip WY, Yeung LS, Choi KC, Chair SY, Dennis CL. Translation and Validation of the Hong Kong Chinese Version of the Breastfeeding Self-Efficacy Scale-Short Form. Res Nurs Health 2012; 35(5):450-459.

30. Victora CG, Araújo CLP, Menezes AMB, Hallal PC, Vieira MF, Neutzling MB. Methodological aspects of the 1993 Pelotas (Brazil) birth cohort study. Rev Saude Publica 2006; 40(1):39-46.

31. Burns PB. Os níveis de evidência e seu papel na medicina baseada em evidências. Plast Reconstr Surg 2010; 128:305-310.

Artigo apresentado em 23/03/2016

Aprovado em 25/10/2016

Versão final apresentada em 27/10/2016 
